MINI REVIEW

\title{
Nuclear Localization of Apolipoprotein E4: A New Trick for an Old Protein
}

\author{
Troy T Rohn* and Zachary D Moore \\ Department of Biological Sciences, Boise State University, USA
}

*Corresponding author: Troy T Rohn, Ph.D., Department of Biological Sciences, Boise State University, 1910 University Drive, Boise, ID 83725, USA, Tel: 208-426-2396, Fax: 208-426-1040, E-mail: trohn@boisestate.edu

\begin{abstract}
One of the most important genetic risk factors for late-onset Alzheimer's Disease (AD) is harboring the ApoE4 allele. Much is known regarding the functions of the ApoE4 protein including cholesterol transport in the CNS and a critical role in clearing beta-amyloid deposits in the AD brain. However, recent studies demonstrating the nuclear localization suggest a novel function beyond the classical known actions of ApoE4. The purpose of the current review is to examine how this secreted protein traffics to the nucleus and to discuss possible outcomes of nuclear localization in the CNS. It is suggested that proteolytic fragmentation of ApoE4 is a key step leading to nuclear localization and the outcome of this event is to initiate transcription of various genes involved in inflammation and cell death. Therefore, the nuclear localization and induction of gene expression may provide a link between harboring the ApoE4 allele and enhanced dementia risk observed in $A D$.
\end{abstract}

\section{Keywords}

Apolipoprotein E4 (ApoE4), Proteolysis, Alzheimer's disease, Nucleus, Transcription, Dementia, Receptor-mediated endocytosis, LDL receptor-related protein

\section{Apolipoprotein E Structure and Function in the CNS}

Human ApoE is polymorphic with three major isoforms, ApoE2, ApoE3, and ApoE4, which differ by single amino acid substitutions involving cysteine-arginine replacements at positions 112 and 158 [1]. ApoE is synthesized as a precursor protein consisting of 317 amino acids containing an 18 amino acid leader sequence. Cleavage of this linker sequence gives the mature form of ApoE consisting of 299 amino acids and a molecular weight of $34 \mathrm{kDa}$ [2]. Structurally, ApoE consists of two major domains that are $\mathrm{N}$-terminal $(20 \mathrm{kDa})$ and $\mathrm{C}$-ter- minal (10 kDa), separated by a short hinge region [3]. The hinge region of ApoE contains multiple protease sensitive sites and evidence now suggests that ApoE4 is much more susceptible to proteolysis than E3 or E2 and this in fact may link the enhanced risk of harboring ApoE4 to dementia observed in AD (see [4] for recent review).

In the CNS, ApoE is synthesized and secreted by astrocytes [5] and, to a lesser extent, by microglia [6], oligodendrocytes [7], and neurons following injury [8]. Functionally, ApoE is a major cholesterol carrier that supports lipid transport and injury repair in the brain. In this manner, ApoE delivers cholesterol to neurons via several different $A p o E$ receptors that make up collectively, the Low-Density Lipoprotein Receptor (LDLR) family $[9,10]$. It has been proposed that because ApoE is the major cholesterol transporter in the brain (see below) and therefore is essential for synaptogenesis in neurons, then ApoE-isoform-dependent differences in these processes may negatively impact synaptic plasticity or recovery of neurons from neurodegeneration as might occur in $A D[11,12]$.

\section{ApoE4 as a Risk Factor for Alzheimer's Disease}

Human ApoE is polymorphic with three major isoforms, ApoE2, ApoE3, and ApoE4, which differ by single amino acid substitutions involving cysteine-arginine replacements at positions 112 and 158 [1]. The frequency of the three polymorphic alleles is $8.4 \%$ for $E 2,77.9 \%$ for E3 and $13.7 \%$ for E4 [13]. Harboring the ApoE3 allele is believed to neither increase nor decrease one's risk of $A D$, while having the $E 2$ form may decrease one's risk. In contrast, inheritance of one copy of the ApoE4 allele

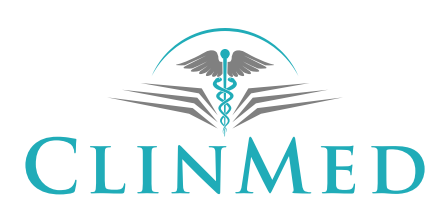

INTERNATIONAL LIBRARY

Citation: Rohn TT, Moore ZD (2017) Nuclear Localization of Apolipoprotein E4: A New Trick for an Old Protein. Int J Neurol Neurother 4:067. doi.org/10.23937/2378-3001/1410067

Received: May 23, 2016: Accepted: July 29, 2017: Published: July 31, 2017

Copyright: (C) 2017 Rohn TT, et al. This is an open-access article distributed under the terms of the Creative Commons Attribution License, which permits unrestricted use, distribution, and reproduction in any medium, provided the original author and source are credited. 
increases disease risk fourfold, while two copies raises the risk tenfold [14]. It is noteworthy that $65-80 \%$ of all AD patients have at least one ApoE4 allele [13,15]. How the ApoE4 protein enhances dementia risk has been the subject of intense study and the prevailing view is
ApoE4 is less effective in promoting the clearance of beta-amyloid deposits. Thus, both pathological and neuroimaging studies have indicated that beta-amyloid deposition occurs to a greater extent and earlier in the disease process in ApoE4 carriers [16-18].

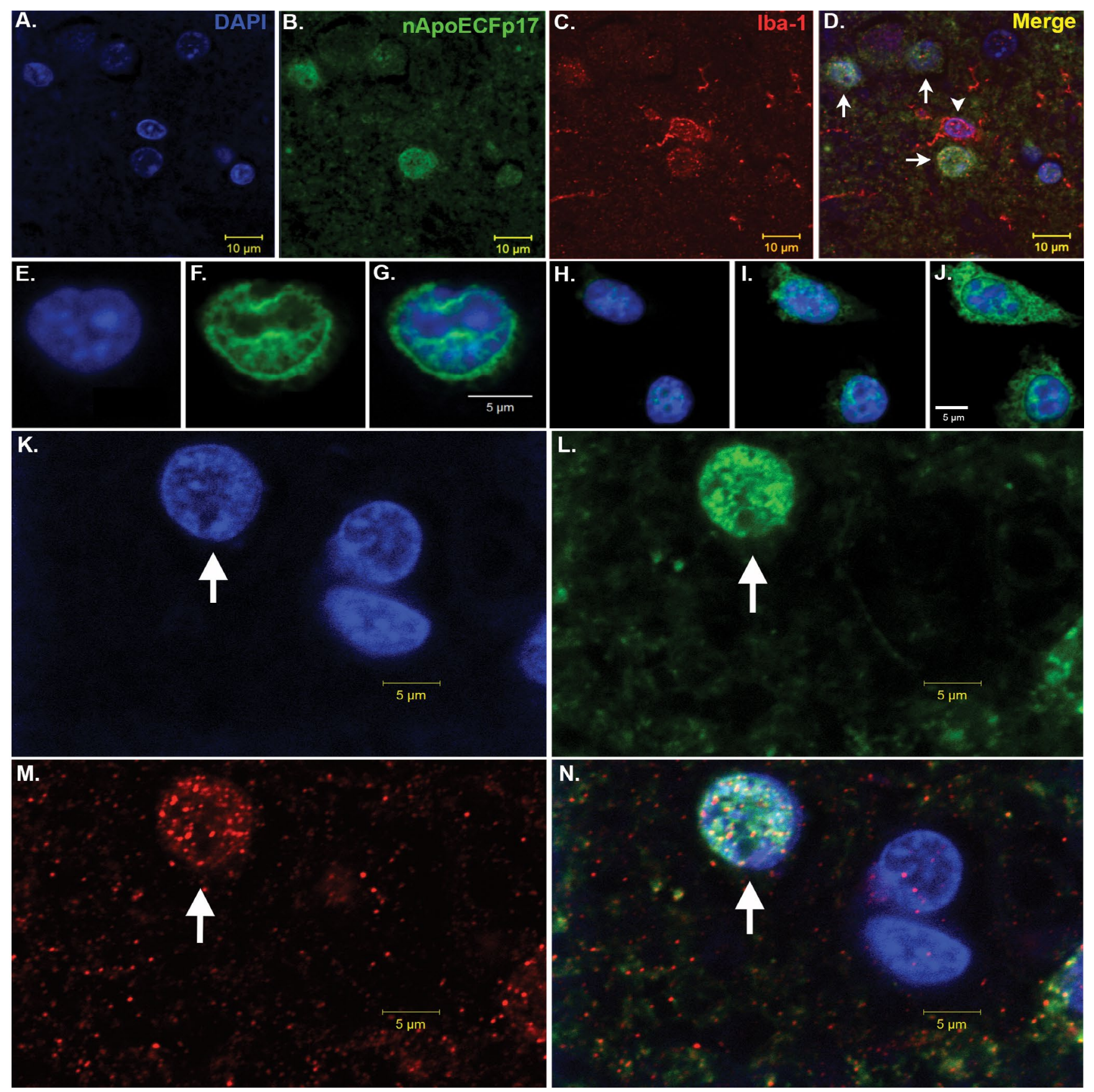

Figure 1: Nuclear localization of an amino-terminal fragment of ApoE within microglia of the AD brain.

(A-D) Representative images from confocal immunofluorescence in AD utilizing the nuclear stain, DAPI, (blue, A), nApoECFp17, an antibody that specifically detects a $17 \mathrm{kDa}$ amino-terminal fragment of ApoE4 (green, B), the microglial specific marker, Iba1 (red, C), with the overlap image shown in (D). Note the colocalization of the two markers with DAPI in Panel D (arrows), however there was one Iba1-labeled microglia that did not label with the nApoECFp17 antibody (arrowhead); (E-J) Two representative sets of images showing nuclear localization of in the microglial cell line, BV2, following exogenous treatment with the ApoE4 $4_{1-151}$ fragment. BV2 microglial cells were placed on glass chamber slides in normal growth media and treated for 24 hours with the His-tagged ApoE4 $4_{1-151}$ fragment $(50 \mu \mathrm{g} / \mathrm{ml})$. Following treatment, cells were fixed and immunocytochemistry was carried out using an anti-His rabbit secondary antibody. Double-label immunofluorescence confocal z-stacks were acquired with an anti-His antibody to detect ApoE4 ${ }_{1-151}$ (green, Panels F and I) together with DAPI (blue, E and H). The merged images indicated the strong nuclear and cytoplasmic presence of the amino-terminal fragment following extracellular incubation of BV2 cells (Panels $\mathrm{G}$ and J); (K-N) Representative images from confocal immunofluorescence in the human AD brain utilizing the nuclear stain, DAPI, (K), nApoECFp17 (L), the microglial specific marker, Iba1 (M), with the overlap image shown in (N). Note the strong nuclear localization of the nApoECFp17 antibody (arrow) within labeled microglia. 
The exact mechanism by which ApoE enhances clearance of beta-amyloid plaque loads is unclear [19-21]. It has been proposed that ApoE can bind to beta-amyloid and undergo endocytosis via the astrocyte low-density Lipoprotein Receptor-Related Protein (LRP) [22,23]. A recent study has demonstrated a differential regulation of beta-amyloid clearance by isoforms of $A p o E$, with ApoE3 being more efficient in this process than ApoE4 [24]. Therefore, harboring the ApoE4 allele may lead to a decreased ability to clear beta-amyloid deposits and accelerate the disease process in AD. An alternative hypothesis is based on a greater propensity of ApoE4 to undergo proteolysis and potential loss of function as compared to ApoE3 or E2 [4]. In this regard, ApoE4 is more susceptible to proteolysis compared to ApoE3 and E2 [25] and ApoE4 fragments (14-20 kDa) have been identified in the $A D$ brain [26]. Further support of this hypothesis came from Bien-Ly, et al. who crossed an AD mouse model with mice that express C-terminal-truncated ApoE4 and showed these mice had a lower affinity for beta-amyloid and a reduced ability to clear beta-amyloid [27].

\section{Nuclear Localization of ApoE4}

Our lab was interested in determining whether proteolysis of ApoE4 was an important event occurring in $A D$ and to address this, we synthesized a site-directed cleavage antibody that specifically recognized an amino-terminal fragment of $17 \mathrm{kDa}$ (p17) following cleavage after D151 of the mature, full-length form of ApoE4 [28]. This antibody, which we termed the amino-terminal ApoE4 cleavage fragment antibody (nApoECFp17 antibody) was highly specific for this fragment following cleavage of full-length ApoE4 with collagenase or MMP9 and showed no immunoreactivity to the full-length, $34 \mathrm{kDa}$ form of the protein [28]. In situ, we demonstrated widespread labeling of this antibody in the AD brain and most surprising, strong localization within microglia (Figure 1). We further showed that a recombinant produced fragment of ApoE4 ${ }_{1-151}$ was taken up by the microglia cell line, BV2, following extracellular treatment and trafficked to the nucleus causing significant toxicity. Interesting, an identical ApoE3 ${ }_{1-151}$ fragment was used as a comparison and although this fragment was taken up into the cytoplasm, no nuclear localization was evident and furthermore, there was no evidence for cytotoxicity [28]. The only difference between these two fragments was the presence of a cysteine residue at position 112 for the ApoE3 ${ }_{1-151}$ fragment in lieu of an arginine in ApoE4 ${ }_{1-151}$. This suggested that nuclear localization of ApoE4 $4_{1-151}$ and eventual cell death could be linked events. Taken together, our results shed light on a potential novel pathway for ApoE4 whereby localization within the nucleus may lead to gene expression of cell death pathways. Figure 2 summarizes a putative pathway of nuclear localization of an amino-terminal fragment of ApoE4 leading to gene expression and cell death.
Structural Differences in ApoE4 Versus ApoE3

\section{Amino-Terminal Fragments}

Our results supported the nuclear localization and cytotoxicity of ApoE4 ${ }_{1-151}$ as compared to ApoE3 ${ }_{1-151}$ ' even though these fragments only differ by a single amino acid. What could account for such a difference? Given that the substitution responsible is only one residue, there may be structural or functional variation between the two amino-terminal fragments with consequential downstream effects. Current predictive modeling techniques can provide great insight into the inherent properties of proteins, and the potential resulting interactions. One such program, the MPI Bioinformatics Toolkit, begins with sequence alignment drawing data from two global protein databases that include RCSB PDB (Research Collaboratory for Structural Bioinformatics Protein Data Bank) and SCOPe (Structural Classification of Proteins-extended) to then assign interspecies proteome homology to an input amino acid sequence. The three-dimensional modeling it generates is based on protein structures that have been determined through X-ray crystallography, NMR spectroscopy, and cryo-electron microscopy [29]. With this data, an algorithm cross-references the protein database information to yield a file containing the predicted tertiary structure of the protein that may be put into any visualization software that recognizes .pdb files. For the images generated in Figure 3, Visual Molecular Dynamics (VMD) was used to visualize the structures [30]. In the case of the two sequences of the 151 amino acid $\mathrm{N}$-terminal fragments of ApoE3 and ApoE4 entered in to the MPI toolkit, $100 \%$ matches to homologous proteins contained within the databanks were found. The proteins bearing PDB identification numbers $1 N F N$ and 1GS9 were used as templates for ApoE3 and ApoE4, respectively, to generate models (Verderame, J.R., Kantardjieff, K., Segelke, B., Weisgraber, K., Rupp, B., 2003; Apolipoprotein E4, 22K domain. PDBID: 1 GS9. DOI: 10.2210/pdb1gs9/pdb. Rupp, B., Parkin, S., 1997; Apolipoprotein E3 (ApoE3). PDBID: 1NFN. DOI: 10.2210/ $\mathrm{pdb} 1 \mathrm{nfn} / \mathrm{pdb}$ ). At first glance the secondary structure of either of ApoE3 ${ }_{1-151}$ and ApoE4 ${ }_{1-151}$ amino-terminal fragments appears similar to full-length ApoE that is both fragment and full-length forms adopt predominantly alpha-helical secondary structure. However, examining the tertiary differences between ApoE3 ${ }_{1-151}$ and ApoE4 ${ }_{1}$. ${ }_{151}$ amino-terminal fragments appears to reveal subtle differences: Although the ApoE3 ${ }_{1-151}$ and ApoE4 ${ }_{1-151}$ amino-terminal fragments differ by only a C112R substitution, the tertiary structure of the $\mathrm{E} 4$ fragment of the protein appears to have taken on an altered tertiary structure based on modeling predictions (Figure 3 ). As seen in Figure 3B, the first 24 residues originating from the $\mathrm{N}$-terminal of ApoE4 ${ }_{1-151}$ do not take on any secondary structure, instead producing a potential "hook" region 


\section{Trafficking of an amino-terminal fragment of ApoE to the nucleus}

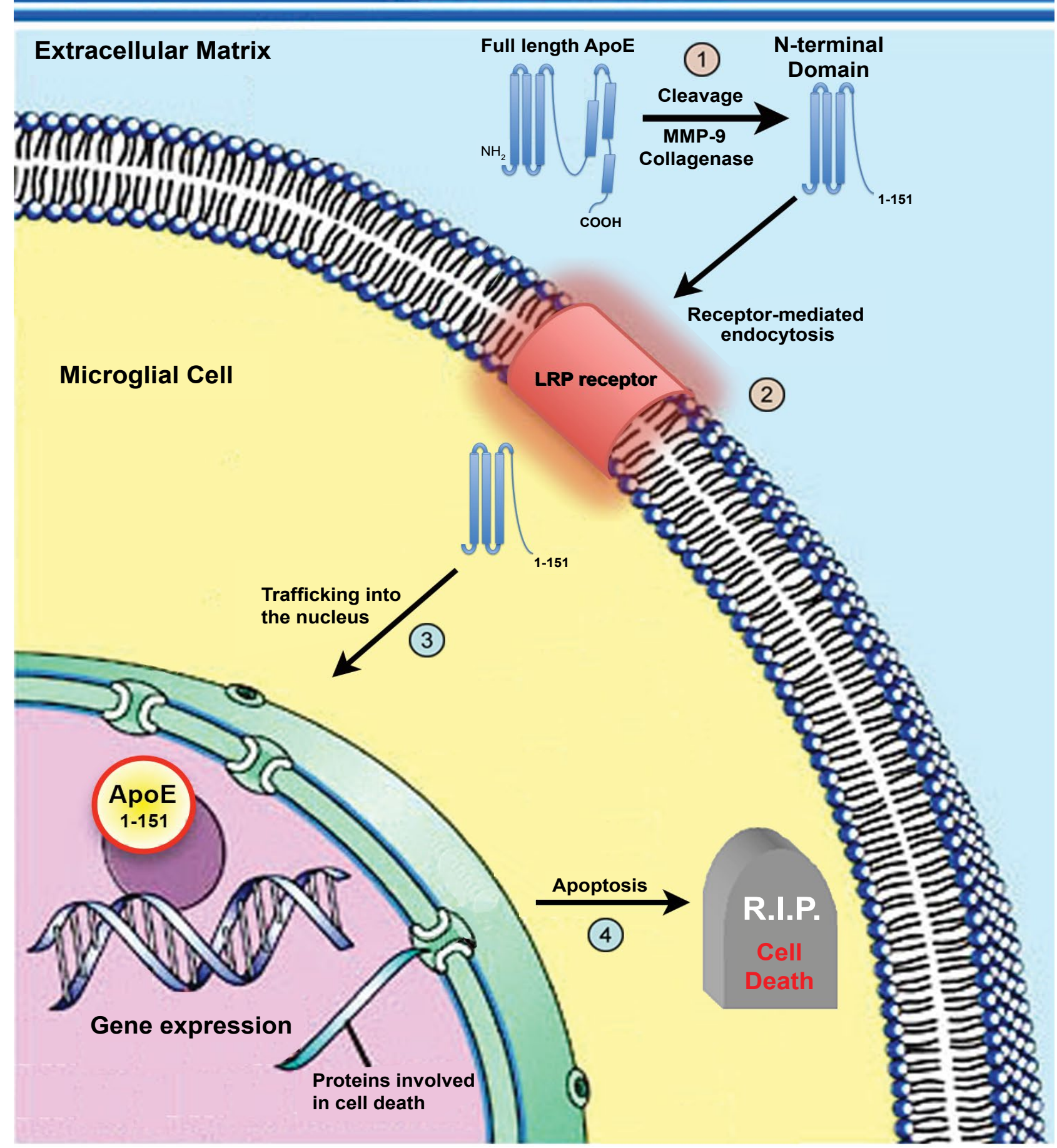

Figure 2: Trafficking of an amino-terminal fragment of ApoE4 to the nucleus.

(1) ApoE4 may promote the pathogenesis underlying AD following cleavage in the extracellular compartment by collagenase or MMP-9; (2) The generation of this fragment by then lead to specific uptake into glial cells such as microglia, through the LRP receptor via Receptor-Mediated Endocytosis (RME); (3) This fragment then traffics to the nucleus and due to its' small size, may passively diffuse through nuclear pore complexes unassisted; (4) Once inside the nucleus, this amino-terminal fragment of ApoE4 may act as a transcription factor leading to the expression of genes that promote cell death or enhance inflammation. In addition to this scheme involving an amino-terminal fragment of ApoE4 generated extracellularly, a similar pathway may be involved for full-length ApoE4 cleaved by unknown proteases within glial cells that normally express ApoE. Cellular entry through RME would lead to residence of ApoE fragments within endosomes and eventually lysosomes (not shown). How ApoE could escape degradation once in lysosomes is currently not known.

that extends outward from the fragment. In contrast, in the ApoE3 ${ }_{1-151}$ fragment, this region adopts a predictive alpha-helical structural that remains folded (Figure $3 A)$. It is possible that this "hook" domain of ApoE4 ${ }_{1-151}$ is the difference that allows for nuclear localization and subsequent toxicity. Although speculative at this point, these potential structural differences could provide impetus for future studies. 

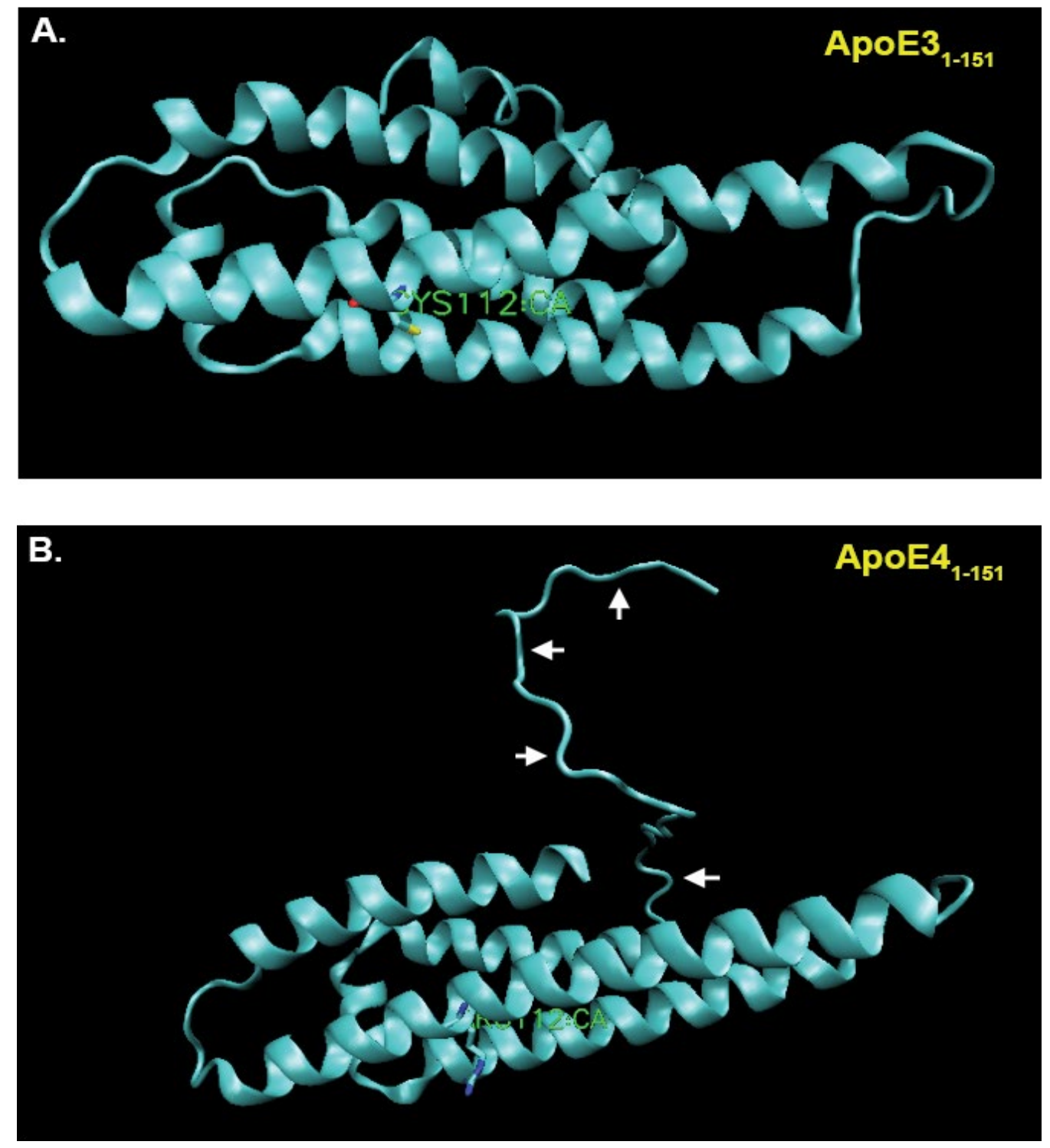

Figure 3: Predictive structural modeling of amino-terminal fragments of ApoE3 and E4.

(A) VMD representation of the MPI-predicted structure of the aminApoE3 ${ }_{1-151}$ with listed cysteine 112 residue shown in green. Note alpha-helical secondary structural predicted by this amino acid sequence; (B) VMD representation of the MPI-predicted structure of the aminApoE4 ${ }_{1-151}$ with listed arginine 112 residue shown in green. In this case, note the "hook" region of the amino-terminus that extends outward from the amino-terminal fragment (arrows).

\section{Other Studies Supporting Nuclear Localization of ApoE}

In addition to our findings several previous studies have also documented the nuclear localization of the ApoE protein. For example, recently, Theendakara, et al. demonstrated a role for ApoE as a DNA binding protein and transcriptional regulator of multiple genes [31]. Using a combination of neural cell lines, skin fibroblasts from $A D$ patients, and ApoE targeted replacement mouse brains, the authors eloquently showed nuclear localization of ApoE4 and binding to double-stranded DNA with high affinity that was in the range of known transcription factors ( 10 $\mathrm{nM}$ ) [31]. A key finding in this study was evidence demonstrating direct binding of ApoE4 and E3 to the SirT1 promoter region [31]. Moreover, the authors were able to identify 76 genes activated by ApoE4 and included among them genes involved in programmed cell death as well as three genes linked to inflammation [31]. Gene expression of proteins involved in programmed cell death would support our findings showing significant cytotoxicity following nuclear localization of ApoE $4_{1-151}$ in BV2 cells [28]. In terms of the transcriptional role of ApoE4 fragments versus full-length ApoE4, it is unknown whether different genes will be regulated. However, it is important to note that in the study by Theendakara, et al., the authors expressed full-length ApoE4 in cells, but did not actually determine whether the transcriptional regulation in their cell model was occurring by full-length ApoE4 or ApoE4 that may have been proteolyses in the cytoplasm by an unknown protease. Further studies will be necessary to see if there are different patterns of gene expression elicited between full-length and fragmented ApoE4.

Levros, et al. demonstrated the binding and repressive activities of ApoE4 and E3 on the human ApoD 
promoter in hepatic and glioblastoma cell lines. In addition, over expression of ApoE3 and E4 isoforms but not ApoE2 significantly inhibited the ApoD promoter activity in $\mathrm{U} 87$ cells suggesting that ApoE can regulate ApoD gene expression [32]. Taken together, these studies support the hypothesis that ApoE can possibly function as a transcription factor, highlighting a novel role for this protein.

Other studies have also supported the nuclear localization of ApoE including Kim, et al. who used Green Fluorescent (GFP) fusion proteins to demonstrated that all three ApoE isoforms could be detected in the nucleus of $\mathrm{CHO}$ cells [33]. Interesting, in this study the level of ApoE-GFP in the nucleus increased following serum starvation, a cellular stress signal [33]. In a parallel study, Quinn, et al. demonstrated nuclear pools of ApoE in human fibroblasts under conditions of serum-starvation-induced growth arrest [34]. The first documented nuclear localization of ApoE came from Panin, et al. who showed immunoreactivity in various rat tissue cells [35]. The authors concluded that ApoE could be involved in the regulation of transcriptional activity of chromatin.

\section{Mechanism of Nuclear Trafficking of ApoE}

A corollary to the nuclear localization of ApoE is how it traffics to the nucleus. Our previous results uncovered a potentially novel mechanism by which ApoE4 is cleaved by MMP-9, and the subsequent amino-terminal fragment is taken up by cells and traffics to the nucleus [28]. Examination of the sequence of the immunogenic peptide of ApoE4 used to synthesize the nApoECF antibody indicates the epitope is near position D151 of the full-length ApoE protein. Cleavage by MMP-9 after this aspartic residue would generate an approximate $17 \mathrm{kDa}$ amino-terminal fragment. Interesting, the ApoE protein has several domains including the $\mathrm{C}$-terminal domain that is known to bind to cholesterol and beta-amyloid, an amino-terminal domain that primarily includes the LDL receptor-binding region (residues 134-150), and a hinge region connecting the two domains that contains a number of putative proteolytic cleavage sites [36,37]. Coincidently, the cleavage site recognized by the $n A$ poECF antibody coincides directly with the low-density Lipoprotein Receptor Related Protein (LRP)-binding region. Therefore, we hypothesize that cleavage of fulllength ApoE4 at position 151 generates an amino-terminal fragment that may interact with high affinity to LRP, is taken up through receptor-mediated endocytosis, traffics to the nucleus, and thereby alters gene expression. We hypothesize that it is the LRP receptor expressed on the cell surface of microglia that is responsible for mediating the uptake of $A_{p o E_{1-151}}$ due to the fact that LRP is the major receptor for lipid-poor forms of ApoE [38]. The lipid-binding region of ApoE is located within the C-terminal domain (residues 244-272) [37], and thus $\mathrm{ApoE}_{1-151}$ by lacking this domain would be lipid free. In addition, the LRP/LDL receptor-binding domain of ApoE (residues 134-150) [36] corresponds exactly to the C-terminal end of $\mathrm{ApoE}_{1-151}$ to which our nApoECF antibody recognizes and labels nuclei in the $A D$ brain. Therefore, we hypothesize that this fragment may bind with high affinity to LRP and is readily taken up by cells expressing this receptor (Figure 2). However, one problem with this hypothesis is the mechanism by with ApoE amino-terminal fragments can escape the endosomal pathway and degradation by specific enzymes in the lysosome is currently unclear. This question will require further investigation.

Once residing in the cytoplasm the next question is how does ApoE traffic to the nucleus? For full-length ApoE having a molecular weight of $34 \mathrm{kDa}$ would suggest that perhaps it could passively diffuse through nuclear pore complexes unassisted. Previous studies have shown that proteins greater than $50 \mathrm{kDa}$ require an active transport pathway largely mediated by importins $[39,40]$. Additionally, ApoE could be transported into the nucleus by binding to specific nuclear proteins through their weak polybasic domains that could serve as potential nuclear signaling sequences [32,33]. Again, further studies will be necessary to determine the exact mechanism of nuclear penetration.

\section{Concluding Remarks}

Harboring the ApoE4 allele represents the most significant genetic risk factor for late-onset AD. For a protein with fairly innocuous actions in terms of it's' ability to shuttle cholesterol around in the CNS, how inheriting this gene leads to an increase in dementia risk has been the subject of intense study. Our current understanding of how this gene translates to enhance risk appears to revolve around, at least in part, beta-amyloid clearance and enhanced proteolysis of ApoE4 leading to a loss of function. However, recent studies have shed light on a potential new mechanism by which ApoE4 may be linked to AD. Proteolysis of ApoE4, which occurs to a greater frequency than with ApoE2 or E3, produces an amino-terminal fragment that is taken up by neurons and glial cells, traffics to the nucleus, and promotes cell death by activation of gene transcription. In this manner, ApoE4 and associated fragments may be serving as transcription factors leading to the expression of genes involved in programmed cell death and inflammation. These findings suggest a novel role for ApoE4 beyond its' classical actions related to lipid trafficking in the CNS. Future studies should help confirm this potential novel role for ApoE4 and perhaps lead to a better understanding as to why inheritance of this gene enhances AD risk.

\section{Acknowledgements}

This work was funded by National Institutes of Health Grant 1R15AG042781-01A1. Funding was also supported by the KO Alzheimer's Dementia Foundation (Boise, ID). The project described was supported by Institutional Development Awards (IDeA) from the National 
Institute of General Medical Sciences of the National Institutes of Health under Grants \#P20GM103408 and P20GM109095. The funders had no role in study design, data collection and analysis, decision to publish, or preparation of the manuscript.

\section{References}

1. Weisgraber KH, Rall SC, Mahley RW (1981) Human E Apoprotein heterogeneity. Cysteine-arginine interchanges in the amino acid sequence of the Apo-E isoforms. J Biol Chem 256: 9077-9083.

2. Mahley RW, Rall SC (2000) Apolipoprotein E: far more than a lipid transport protein. Annu Rev Genomics Hum Genet 1: 507-537.

3. Wetterau JR, Aggerbeck LP, Rall SC, Weisgraber $\mathrm{KH}$ (1988) Human Apolipoprotein E3 in aqueous solution. I. Evidence for two structural domains. J Biol Chem 263: 6240-6248.

4. Rohn TT (2013) Proteolytic cleavage of Apolipoprotein e4 as the keystone for the heightened risk associated with Alzheimer's disease. Int J Mol Sci 14: 14908-14922.

5. Boyles JK, Pitas RE, Wilson E, Mahley RW, Taylor JM (1985) Apolipoprotein E associated with astrocytic glia of the central nervous system and with nonmyelinating glia of the peripheral nervous system. J Clin Invest 76: 1501-1513.

6. Qin S, Colin C, Hinners I, Gervais A, Cheret C, et al. (2006) System Xc- and Apolipoprotein E expressed by microglia have opposite effects on the neurotoxicity of amyloid-beta peptide 1-40. J Neurosci 26: 3345-3356.

7. Stoll G, Meuller HW, Trapp BD, Griffin JW (1989) Oligodendrocytes but not astrocytes express Apolipoprotein $E$ after injury of rat optic nerve. Glia 2: 170-176.

8. Xu Q, Bernardo A, Walker D, Kanegawa T, Mahley RW, et al. (2006) Profile and regulation of Apolipoprotein E (ApoE) expression in the CNS in mice with targeting of green fluorescent protein gene to the ApoE locus. J Neurosci 26: 4985-4994.

9. Pitas RE, Boyles JK, Lee SH, Hui D, Weisgraber KH (1987) Lipoproteins and their receptors in the central nervous system. Characterization of the lipoproteins in cerebrospinal fluid and identification of Apolipoprotein $B, E(L D L)$ receptors in the brain. J Biol Chem 262: 14352-14360.

10. Michikawa M, Fan QW, Isobe I, Yanagisawa K (2000) Apolipoprotein $E$ exhibits isoform-specific promotion of lipid efflux from astrocytes and neurons in culture. $J$ Neurochem 74: 1008-1016.

11. Mauch DH, Nagler K, Schumacher S, Goritz C, Muller EC, et al. (2001) CNS synaptogenesis promoted by glia-derived cholesterol. Science 294: 1354-1357.

12. Hayashi H, Campenot RB, Vance DE, Vance JE (2004) Glial lipoproteins stimulate axon growth of central nervous system neurons in compartmented cultures. J Biol Chem 279: 14009-14015.

13. Farrer LA, Cupples LA, Haines JL, Hyman B, Kukull WA, et al. (1997) Effects of age, sex, and ethnicity on the association between Apolipoprotein $E$ genotype and Alzheimer disease. A meta-analysis. ApoE and Alzheimer Disease Meta Analysis Consortium. JAMA 278: 1349-1356.

14. Eisenstein M (2011) Genetics: finding risk factors. Nature 475: S20-S22.

15. Saunders AM, Strittmatter WJ, Schmechel D, George-Hys- lop PH, Pericak-Vance MA, et al. (1993) Association of Apolipoprotein $\mathrm{E}$ allele epsilon 4 with late-onset familial and sporadic Alzheimer's disease. Neurol 43: 1467-1472.

16. Rebeck GW, Reiter JS, Strickland DK, Hyman BT (1993) Apolipoprotein $\mathrm{E}$ in sporadic Alzheimer's disease: allelic variation and receptor interactions. Neuron 11: 575-580.

17. Morris JC, Roe CM, Xiong C, Fagan AM, Goate AM, et al. (2010) ApoE predicts amyloid-beta but not tau Alzheimer pathology in cognitively normal aging. Ann Neurol 67: 122131.

18. Reiman EM, Chen K, Liu X, Bandy D, Yu M, et al. (2009) Fibrillar amyloid-beta burden in cognitively normal people at 3 levels of genetic risk for Alzheimer's disease. Proc Natl Acad Sci USA 106: 6820-6825.

19. Veeraraghavalu K, Zhang C, Miller S, Hefendehl JK, Rajapaksha TW, et al. (2013) Comment on "ApoE-Directed Therapeutics Rapidly Clear beta-Amyloid and Reverse Deficits in AD Mouse Models". Science 340: 924.

20. Tesseur I, Lo AC, Roberfroid A, Dietvorst S, Van Broeck B, et al. (2013) Comment on "ApoE-directed therapeutics rapidly clear beta-amyloid and reverse deficits in $A D$ mouse models". Science 340: 924e.

21. Verghese PB, Castellano JM, Garai K, Wang $Y$, Jiang $H$, et al. (2013) ApoE influences amyloid-beta (Abeta) clearance despite minimal ApoE/Abeta association in physiological conditions. Proc Natl Acad Sci U S A 110: E1807-E1816.

22. Gylys KH, Fein JA, Tan AM, Cole GM (2003) Apolipoprotein $E$ enhances uptake of soluble but not aggregated amyloid-beta protein into synaptic terminals. J Neurochem 84 : 1442-1451.

23. Zerbinatti CV, Wahrle SE, Kim H, Cam JA, Bales K, et al. (2006) Apolipoprotein $E$ and low density lipoprotein receptor-related protein facilitate intraneuronal Abeta42 accumulation in amyloid model mice. J Biol Chem 281: 36180-36186.

24. Li J, Kanekiyo T, Shinohara M, Zhang Y, LaDu MJ, et al. (2012) Differential regulation of amyloid-beta endocytic trafficking and lysosomal degradation by Apolipoprotein $\mathrm{E}$ isoforms. J Biol Chem 287: 44593-44601.

25. Harris FM, Brecht WJ, Xu Q, Tesseur I, Kekonius L, et al. (2003) Carboxyl-terminal-truncated Apolipoprotein E4 causes Alzheimer's disease-like neurodegeneration and behavioral deficits in transgenic mice. Proc Natl Acad Sci USA 100: 10966-10971.

26. Cho HS, Hyman BT, Greenberg SM, Rebeck GW (2001) Quantitation of ApoE domains in Alzheimer disease brain suggests a role for ApoE in Abeta aggregation. J Neuropathol Exp Neurol 60: 342-349.

27. Bien-Ly N, Andrews-Zwilling Y, Xu Q, Bernardo A, Wang C, et al. (2011) C-terminal-truncated Apolipoprotein (Apo) E4 inefficiently clears amyloid-beta (Abeta) and acts in concert with Abeta to elicit neuronal and behavioral deficits in mice. Proc Natl Acad Sci USA 108: 4236-4241.

28. Love JE, Day RJ, Gause JW, Brown RJ, Xinzhu P, et al. (2017) Nuclear uptake of an amino-terminal fragment of Apolipoprotein E4 promotes cell death and localizes within microglia of the Alzheimer's disease brain. Int J Physiol Pathophysiol Pharmacol 9: 40-57.

29. Berman HM, Westbrook J, Feng Z, Gilliland G, Bhat TN, et al. (2000) The Protein Data Bank. Nucleic Acids Res 28: 235-242.

30. Humphrey W, Dalke A, Schulten K (1996) VMD: visual molecular dynamics. J Mol Graph 14: 33-38, 27-28. 
31. Theendakara V, Peters-Libeu CA, Spilman P, Poksay KS, Bredesen DE, et al. (2016) Direct Transcriptional Effects of Apolipoprotein E. J Neurosci 36: 685-700.

32. Levros LC Jr, Labrie M, Charfi C, Rassart E (2013) Binding and repressive activities of Apolipoprotein E3 and E4 isoforms on the human ApoD promoter. Mol Neurobiol 48: 669-680.

33. Kim WS, Elliott DA, Kockx M, Kritharides L, Rye KA, et al. (2008) Analysis of Apolipoprotein E nuclear localization using green fluorescent protein and biotinylation approaches. Biochem J 409: 701-709.

34. Quinn CM, Kagedal K, Terman A, Stroikin U, Brunk UT, et al. (2004) Induction of fibroblast Apolipoprotein E expression during Apoptosis, starvation-induced growth arrest and mitosis. Biochem J 378: 753-761.

35. Panin LE, Russkikh GS, Polyakov LM (2000) Detection of Apolipoprotein A-I, B, and E immunoreactivity in the nuclei of various rat tissue cells. Biochemistry (Mosc) 65: 14191423.

36. Mahley RW, Huang Y (2012) Small-molecule structure correctors target abnormal protein structure and function: structure corrector rescue of Apolipoprotein E4-associated neuropathology. J Med Chem 55: 8997-9008.

37. Bu G (2009) Apolipoprotein E and its receptors in Alzheimer's disease: pathways, pathogenesis and therapy. Nat Rev Neurosci 10: 333-344.

38. Narita M, Holtzman DM, Fagan AM, LaDu MJ, Yu L, et al. (2002) Cellular catabolism of lipid poor Apolipoprotein E via cell surface LDL receptor-related protein. J Biochem 132: 743-749.

39. Yoneda $Y$ (2000) Nucleocytoplasmic protein traffic and its significance to cell function. Genes Cells 5: 777-787.

40. Ohno M, Fornerod M, Mattaj IW (1998) Nucleocytoplasmic transport: the last 200 nanometers. Cell 92: 327-336. 